\title{
Manfred Thielen, Angela von Arnim, Anna Willach- Holzapfel (Hrsg.): Lebenszyklen - Körperrhythmen. Körperpsychotherapie über die Lebensspanne
}

\author{
Psychosozial, 2018, Gießen, 385 Seiten, 39,90€ (D)
}

$\mathrm{D}$ ieses Buch gibt einen vielfältigen und fundierten Überblick zur aktuellen Körperpsychotherapie mit einem Einblick in das Verständnis und die Methodik zu zentralen Themen des Menschen über die gesamte Lebensspanne hinweg. Drei einleitende Beiträge bilden das inhaltliche Fundament für die vielfältigen Artikel, die sich dann ausgewählten Themen der Lebenszyklen widmen - Schwangerschaft, Geburt, Eltern-Baby-Therapien, Kindheit und Jugend, die Bedeutung von Sexualität und geschlechtlicher Identität sowie schließlich der Umgang mit dem Älterwerden, mit Krankheit und Sterblichkeit. Ein abschließender Schwerpunkt widmet sich speziellen Fragen der Körperpsychotherapie, ihr Verständnis als Humanistische Psychotherapie, spezifisch therapeutische Zugangswege sowie die Reflexion zu Wirkfaktoren. Es wird mit diesen Beiträgen also ein großer inhaltlicher Bogen geschlagen, so dass man einen umfassenden Einblick zum derzeitigen Stand der Körperpsychotherapie gewinnt. Dies ist insofern spannend, da die Köperpsychotherapie aus einer Vielzahl einzelner Richtungen besteht, die sich nach Thielen (S.282) drei Grundorientierungen zuordnen lassen: neoreichianische Richtungen (Bioenergetik, Biodynamik, Biosynthese u.a.), wahrnehmungsbezogene Richtungen (Konzentrative Bewegungstherapie, Funktionelle Entspannung, Integrative Leib- und Bewegungstherapie u. a.) sowie analytische Körperpsychotherapie.

Von der Beobachtung geleitet, dass Lebenszyklen sich in Rhythmen gestalten, entfaltet die Ärztin Angela von Arnim die Idee, Rhythmus als „das grundlegendste Kommunikationsinstrument zwischen Organismus und Umwelt" (S. 20) zu sehen. Rhythmus wird in ihrem Ver- ständnis der Körpertherapie als Lebensprinzip verstanden, Heilung als „Ermöglichung von ,Rhythmusgesundung““ (S.20). Diese Eigenrhythmen des Menschen sind ontogenetisch häufig gestört, wofür von Arnim Beispiele aufzeigt und hier auch Bezug auf molekularbiologische sowie epigenetische Forschungsergebnisse nimmt. Funktionelle Entspannung kann helfen, den Eigenrhythmus wiederzufinden und im Körpersymptom unterbrochene Rhythmusphänomene sowie dort gebundene Traumatisierungen zu bearbeiten. Sehr bedeutsam erscheint mir ihr Hinweis, hierbei die therapeutische Aufmerksamkeit „etwas weniger auf Techniken und Übungen zur kathartischen Aggressionsabfuhr zu richten“ (S. 42), dafür durch phänomenologische Achtsamkeit das Selbsterleben zu fokussieren, so dass Wachstum möglich werden kann.

In dem anregenden Artikel „Selbst und Struktur" plädiert Ulrich Geuter für ein entwicklungspsychologisches Verständnis, das sich an eine Theorie des Selbst anlehnt. Ihm ist daran gelegen, für die therapeutische Arbeit das erlebende und handelnde Subjekt ins Zentrum der Arbeit zu stellen. Das Selbst wird von Geuter als „erlebte Einheit von Erfahrung“ (S.54), die immer verkörpert und ontologisch gegeben ist, verstanden. Entwicklungspsychologisch verlagert sich der Blick dann auf „die Art und Weise, wie jemand sich und die Welt erlebt und wie seine Selbstsinne Erfahrungen bewerten“ (S.55), wie sich also „Körpererleben und Selbsterleben in den Interaktionserfahrungen der Kindheit herausbilden“ (S. 64). 
Der Psychologe Manfred Thielen, Vorsitzender der DGK, verweist in seinem Artikel „Körperpsychotherapie in den verschiedenen Lebenszyklen" auf die auch gesellschaftliche Verantwortung der (Körper-)Psychotherapie als emanzipatorische Kraft. Es kann nicht nur um eine „schnelle Wiederherstellung der Arbeitsund Anpassungsfähigkeit der PatientInnen“ (S.69) gehen. Körperpsychotherapie habe in ihrem Verhältnis zur neoliberalen Gesellschaft eine kritische Funktion, die auch bereits Wilhelm Reich im Blick hatte. Thielen geht es darum, den Menschen zu befähigen, „für seine weltanschaulichen, ethischen, politischen und persönlichen Ziele, Motive, Bedürfnisse und Gefühle aktiv“ (S. 70) einzutreten. Dies erklärt auch die Zuordnung der Körperpsychotherapie zur Humanistischen Psychotherapie. Differenziert zeigt er die besondere Bedeutung der Körperpsychotherapie und ihrer viel- fältigen Methoden in den Phasen des menschlichen Lebens auf.

Über zwanzig weitere Artikel beinhalten Konzepte körpertherapeutischer Arbeit sowie Reflexionen zu spezifischen Themen. Aufgrund dieser Fülle reißen sie mitunter nur Aspekte an oder spitzen die Analyse pointiert zu. Einen weiteren Gewinn würde ich in der deutlicheren Differenzierung sehen, die im Horizont der Leiblichkeit sich zeigenden Phänomene weniger kategorial, sondern vielmehr existenzial zu verstehen. Insgesamt liefert das Buch einen hervorragenden und anregenden Ein- und Überblick in das Verständnis und die Bedeutung der Körpertherapie für PsychotherapeutInnen aller Schulen.

\section{Dr. Christoph Kolbe}

DOI 10.2378/ ktb2022.arto6d 